\title{
Array-based technology and recommendations for utilization in medical genetics practice for detection of chromosomal abnormalities
}

\author{
Melanie Manning, MD, MS, FACMG, and Louanne Hudgins, MD, FACMG, \\ for the Professional Practice and Guidelines Committee
}

\begin{abstract}
Disclaimer: This guideline is designed primarily as an educational resource for health care providers to help them provide quality medical genetic services. Adherence to this guideline does not necessarily assure a successful medical outcome. This guideline should not be considered inclusive of all proper procedures and tests or exclusive of other procedures and tests that are reasonably directed to obtaining the same results. In determining the propriety of any specific procedure or test, the geneticist should apply his or her own professional judgment to the specific clinical circumstances presented by the individual patient or specimen. It may be prudent, however, to document in the patient's record the rationale for any significant deviation from this guideline.
\end{abstract}

\begin{abstract}
Laboratory evaluation of patients with developmental delay/ intellectual disability, congenital anomalies, and dysmorphic features has changed significantly in the last several years with the introduction of microarray technologies. Using these techniques, a patient's genome can be examined for gains or losses of genetic material too small to be detected by standard G-banded chromosome studies. This increased resolution of microarray technology over conventional cytogenetic analysis allows for identification of chromosomal imbalances with greater precision, accuracy, and technical sensitivity. A variety of array-based platforms are now available for use in clinical practice, and utilization strategies are evolving. Thus, a review of the utility and limitations of these techniques and recommendations regarding present and future application in the clinical setting are presented in this study. Genet Med 2010:12(11):742-745.
\end{abstract}

Key Words: array comparative genomic hybridization, malformations, development disabilities, autism, cytogenetics

aboratory evaluation of patients with developmental delay/ Lintellectual disability (DD/ID), congenital anomalies, and dysmorphic features has changed significantly in the last several years with the introduction of microarray technologies (arraybased comparative genomic hybridization $[\mathrm{CGH}]$ and single nucleotide polymorphism [SNP] array analysis) into the menu of tests available to the practicing clinician. With these techniques, a patient's genome is examined for detection of gains or losses of genetic material that typically are too small to be detectable by standard G-banded chromosome studies. Guide-

From the Departments of Pathology and Pediatrics, Stanford University School of Medicine, Stanford, California.

Melanie Manning, MD, MS, FACMG, Stanford University School of Medicine, 200 Pasteur Drive, H315, Stanford, CA 94305. E-mail: mmanning@stanford.edu.

Disclosure: The authors declare no conflict of interest.

Published online ahead of print October 18, 2010.

DOI: $10.1097 /$ GIM.0b013e3181f8baad lines for the application of array-based technology in the practice of medical genetics were originally published in this journal in 2007. ${ }^{1}$ However, because of the rapidly expanding use of genomic copy number microarrays in the clinical setting, we update the recommendations in this study.

\section{MICROARRAY FOR EVALUATION OF COPY NUMBER VARIATION}

Diagnostic cytogenetic testing has undergone a marked evolution since the introduction of chromosome banding techniques in the late 1960s. ${ }^{2}$ DNA-based techniques such as genomic copy number microarrays (cytogenetic microarrays [CMAs]) are the latest tools available for clinical use. ${ }^{1}$ The initial CGH technology was developed for genome-wide screening for unbalanced rearrangements in a single experiment. ${ }^{1,3-5}$ However, the resolution of this "conventional" $\mathrm{CGH}$ was only 3-10 $\mathrm{Mb}^{4}$ comparable with high-resolution karyotyping, ${ }^{1}$ thus $\mathrm{CGH}$ was further adapted for use on microarrays. ${ }^{6}$ With array CGH, cloned (e.g., bacterial artificial chromosomes [BACs]) or synthesized (e.g., oligonucleotides [oligos]) DNA fragments representing precise chromosomal loci across the genome are immobilized on a glass surface. ${ }^{1,7,8}$ Copy number variants (CNVs) are determined by the differences in hybridization pattern intensities between patient DNA and control DNA. ${ }^{7}$ Hybrid SNP/oligo arrays have been developed for the analysis of CNVs and detection of copy number neutral regions of homozygosity. For these analyses, the patient DNA is labeled and hybridized to the microarray, and the patient results are compared with a well-studied reference DNA. ${ }^{9}$ It should be noted that not all CNVs are pathologic, in that it has been demonstrated that the mean number of benign CNVs per person could be as high as 800 or more. ${ }^{10}$

The resolution and yield of an array is limited by the genomic coverage (the length of and spacing between probes) on the microarray ${ }^{3,11,12}$ and by the specific statistical algorithms used to set the criteria for gains and losses. The increased resolution of microarray technology over conventional cytogenetic analysis allows for identification of chromosomal imbalances with greater precision, accuracy, and technical sensitivity. 


\section{CLINICAL UTILITY}

Rauch et al. ${ }^{13}$ investigated the diagnostic yield of various genetic tests used in the evaluation of patients with unexplained cognitive impairment. In their study, molecular karyotyping would only miss $\sim 0.6 \%$ of cases with disease-causing balanced de novo aberrations. They noted that molecular karyotyping in every patient with cognitive impairment would have the highest diagnostic yield of any single test $(28.9 \%)$ and was, thus, suggested by the authors to be considered a first-tier test. Recently, Miller et al. ${ }^{14}$ reviewed the evidence for utilization of CMA as a first-tier test for the investigation of DD/ID, multiple congenital anomalies, and/or autism spectrum disorders (ASDs). These authors' recommendation for use of CMA as a first-tier test was based on studies of 21,698 patients referred for the above-listed indications, in whom the diagnostic yield was $12.2 \%$ higher than that of a G-banded karyotype. After a review of 36,325 patients with $\mathrm{DD} / \mathrm{ID}$, Hochstenbach et al. ${ }^{15}$ also recommended that CMA be a first-tier test in this group of patients. In their study, a pathogenic anomaly was found in $19 \%$. Shen et al. ${ }^{16}$ specifically investigated the utility of CMA in the investigation of children with ASD. They also provided evidence that analysis should be the first-tier test for children with autism. They found that although abnormalities were found on karyotyping and fragile $\mathrm{X}$ testing $2.23 \%$ and $0.46 \%$ of the time, respectively, a microarray identified deletions or duplications in $18.2 \%$ of patients $(N=848)$, with $7 \%$ of those clearly abnormal.

In addition to the linking of submicroscopic de novo deletions and duplications identified on CMA to the patients' disorders, ${ }^{17,18}$ de novo CNVs have also been implicated in increasing the risk for ASD and point to regions of the genome that may house candidate genes for autism. ${ }^{19,20}$

Additional uses for CMA include the investigation of individuals with physical or cognitive impairment but in whom conventional cytogenetic studies demonstrated an apparently balanced translocation. Subsequent CMA investigation demonstrated that the karyotype was actually unbalanced; several studies have shown that approximately $20 \%$ of individuals with an apparently balanced translocation (de novo or familial) have loss or gain of genetic material as identified by CMA. ${ }^{21,22}$ More recently, CMA has been shown to be valuable in uncovering chromosomal regions of medical importance apart from the original indication of the study. Adam et al. ${ }^{23}$ reported three patients evaluated for DD or dysmorphic features/multiple anomalies who were found by CMA to have microdeletions encompassing known tumor susceptibility genes. In a larger study, Adams et al. ${ }^{24}$ found that $0.18 \%$ of patients with an identified gain or loss of genetic material had the inclusion of a gene associated with a cancer-predisposing condition. Both sets of authors emphasized that information gained from CMA, such as the unexpected finding of tumor susceptibility, can have a direct bearing on the future medical management of patients with DD/multiple congenital anomalies in addition to providing an explanation of the general phenotype.

In addition to identifying copy number variants that affect the number of copies of a particular gene or genes, microarray analysis could theoretically identify genes that are disrupted by breakpoints in the genome. Disruption could be on the basis of interruption of a coding sequence or of a sequence that affects transcription and translation, e.g., sequence changes in a promoter region. Examples include duplications and deletions involving the NRXN1 and CNTN4 genes. ${ }^{25,26}$ Finally, as pointed out by Moeschler. ${ }^{27,28}$ and Saam et al., ${ }^{29}$ an accurate diagnosis for patients will provide the clinician the opportunity to discuss treatment options, prognosis, and recurrence risks as well as to avoid unnecessary future testing.

\section{PLATFORMS}

Although microarray analysis is proficient in characterizing chromosomal imbalances (which ultimately improves patient care), ${ }^{29}$ clinicians ordering the test need to be aware of the different clinical platforms (e.g., BAC versus oligo, targeted versus whole genome, and SNP), the variation in resolution among arrays and the information each provides. For example, many clinicians are unaware that a whole genome oligoarray can detect clinically significant copy number changes missed on a targeted BAC array ${ }^{30}$ or that a SNP array can detect long contiguous stretches of homozygosity that can be associated with uniparental disomy or consanguinity, both of which increase the risk for autosomal recessive conditions.

Array resolution is dependent on the number and types of probes used and how they are distributed across the genome. ${ }^{31}$ $\mathrm{BAC}$ probes are larger than oligonucleotide probes used for oligo and SNP arrays (BACs are $\sim 75,000$ to 150,000 base pairs in length, whereas oligos are usually $\sim 50$ to 60 base pairs long). This translates into reduced breakpoint specificity of copy number abnormalities for the BAC arrays. Higher probe density on oligo arrays allows for copy number evaluation to be based on multiple adjacent probes, enhancing the accuracy of the interpretation. Oligonucleotide array construction tends to have better reproducibility and less batch-to-batch variation than does BAC construction. ${ }^{31}$

SNP microarrays are applications of microarray technology that also provide genome-wide copy number analysis. In addition to copy number changes, SNP arrays are able to detect so-called "copy number neutral" abnormalities such as segmental uniparental disomy and areas of long contiguous stretches of homozygosity that can give rise to disease, congenital anomalies, or cognitive impairment. ${ }^{32,33}$ SNP arrays are increasingly being used in the assessment of cognitive impairment or DD, with or without associated anomalies and are likely to be used in the diagnosis of these conditions. ${ }^{9,11}$

When ordering a CMA, the clinician should be aware of the various platforms currently in use and their limitations. Questioning the laboratory performing the test about coverage of the array in specific regions of interest (e.g., telomeres, $\mathrm{X}$ chromosome, and common microdeletions) is justified. The clinician also should understand what type of follow-up tests will be performed, and on whom, in the event of abnormal results. Further, for deletions and duplications, parental studies (by fluorescence in situ hybridization [FISH] or metaphase preparations, if possible) should be conducted to rule out the presence of a chromosomal rearrangement such as an insertion or inherited duplication. Although rare, for a family in which such a rearrangement is found, recurrence risk can be as high as $50 \%$. With increased utilization of a diagnostic test comes a better appreciation of the range of possible and sometimes unexpected results. This is certainly the case with array CGH and identification of what we now understand to be benign CNVs. An international consortium of more than 75 laboratories has been formed to address questions surrounding array-based testing. The International Standard Cytogenomic Array consortium (https://isca.genetics.emory.edu/iscaBrowser/) is investigating the feasibility of establishing a standardized, universal system of reporting and cataloging $\mathrm{CGH}$ results, both pathologic and benign, to provide the clinician with the most accurate and up-to-date information. ${ }^{14}$ Databases currently available for referencing gene location and function, $\mathrm{CNV}$ listings, and up-to-date 
clinical information for specific abnormalities include the UC Santa Cruz Database (http://www.genome.uscs.edu), the Toronto Database of Genomic Variants (http://projects/tcag.ca/variation/), DECIPHER (http://www.sanger.ac.uk/PostGenomics/decip), and ECARUCA. ${ }^{34}$

Even though CMA technology has greatly improved since it was initially developed, ${ }^{4}$ clinicians ordering these tests must be aware of the limitations that remain. Array CGH cannot identify balanced chromosomal rearrangements, such as translocations or inversions, or differentiate free trisomies from unbalanced Robertsonian translocations. ${ }^{13,35,36}$ Some aneuploidies can be missed, such as XYY if the wrong gender control is used. ${ }^{31}$ Marker chromosomes may also be missed, depending on the size, marker composition, and array coverage of the specific chromosomal region present on the marker. ${ }^{35}$ Detection of mosaicism has been reported, but the accuracy of detecting low levels described by some groups ${ }^{37}$ has been questioned by others. ${ }^{35,36}$ Recently, Scott et al. ${ }^{38}$ suggested that mosaicism for an extra chromosome could be detected at the $10 \%$ level, whereas mosaicism for deletion or duplication of part of a chromosome could be detectable at the $20-30 \%$ level. These findings remain to be replicated by others. Interpretation of the significance of a rare copy number change can be incomplete if parental samples are unavailable for comparison and published data on the CNV are lacking. Finally, triploidy will not be detected by some forms of microarray.

A microarray should not be ordered when a rapid turnaround time is needed (e.g., a STAT newborn analysis), especially if a chromosomal trisomy is suspected. Currently, a STAT G-banded chromosome analysis can be performed within 48 hours. With some array CGH platforms, hybridization alone can take 48 hours. Although technically some arrays may be run in $3-5$ days in some laboratories, analysis and confirmation of results with FISH (development of a unique probe can take weeks) and analysis of parental samples and interpretation may take much longer.

Although microarray is a powerful diagnostic tool for the evaluation of chromosomal copy number changes, its use as a first-tier test may not always be appropriate. For example, conventional karyotyping may be more appropriate when a common aneuploidy (e.g., trisomy 21 , trisomy 18 , or a sex chromosome aneuploidy) is suspected. FISH with a single probe to confirm a suspected diagnosis of a well-described syndrome, such as Williams syndrome, would be a more cost-effective testing methodology. CMA also should not be used in cases of family history of chromosome rearrangement in a phenotypically normal individual or in cases of multiple miscarriages. ${ }^{14}$ Finally, CMA cannot detect low-level mosaicism or, in some arrays, polyploidy.

\section{Recommendations}

1. CMA testing for $\mathrm{CNV}$ is recommended as a first-line test in the initial postnatal evaluation of individuals with the following:

A. Multiple anomalies not specific to a well-delineated genetic syndrome.

B. Apparently nonsyndromic DD/ID.

C. Autism spectrum disorders.

2. Further determination of the use of CMA testing for the evaluation of the child with growth retardation, speech delay, and other less well-studied indications is recommended, particularly by prospective studies and aftermarket analysis.

3. Appropriate follow-up is recommended in cases of chromosome imbalance identified by CMA, to include cyto-
genetic/FISH studies of the patient, parental evaluation, and clinical genetic evaluation and counseling.

\section{REFERENCES}

1. Manning M, Hudgins L. Use of array-based technology in the practice of medical genetics. Genet Med 2007;9:650-653.

2. Trask BJ. Human cytogenetics: 46 chromosomes, 46 years and counting. Nat Rev Genet 2002:3:769-778.

3. Shaffer LG, Beaudet AL, Brothman AR, et al. Microarray analysis for constitutional cytogenetic abnormalities. Genet Med 2007;9:654-662.

4. Pinkel D, Segraves R, Sudar D, et al. High resolution analysis of DNA copy-number variation using comparative genomic hybridization to microarrays. Nat Genet 1998;20:207-211.

5. Bejjani BA, Saleki R, Ballif BC, et al. Use of targeted array-based CGH for the clinical diagnosis of chromosomal imbalances: is less more? Am J Med Genet 2005;134:259-267.

6. Solinas-Toldo S, Lampel S, Stilgenbauer S, et al. Matrix-based comparative genomic hybridization: biochips to screen for genomic imbalances. Genes Chromosomes Cancer 1997;20:399-407.

7. Oostlander AE, Meijer GA, Ylstra B. Microarray-based comparative genomic hybridization and its application in human genetics. Clin Genet 2004;66:488-495

8. Snijders AM, Nowak N, Segraves R, et al. Assembly of microarrays for genome-wide measurements of DNA copy number. Nat Genet 2001;29:263264

9. Bernardini L, Alesi V, Loddo S, et al. High-resolution SNP arrays in mental retardation diagnostics: how much do we gain? Eur J Hum Genet 2010;18: $178-185$.

10. Tyson C, Harvard C, Locker R, et al. Submicroscopic deletions and duplications in individuals with intellectual disability detected by array-CGH Am J Med Genet 2005;139:173-185.

11. Friedman J, Adam S, Arbour L, et al. Detection of pathogenic copy number variants in children with idiopathic intellectual disability using $500 \mathrm{~K} \mathrm{SNP}$ array genomic hybridization. BMC Genomics 2009;10:526.

12. Moeschler JB, Shevell M. Clinical genetic evaluation of the child with mental retardation or developmental delays. Pediatrics 2006;117:23042316.

13. Rauch A, Hoyer J, Guth S, et al. Diagnostic yield of various genetic approaches in patients with unexplained developmental delay or mental retardation. Am J Med Genet 2006;140:2063-2074.

14. Miller DT, Adam MP, Aradhya S, et al. Consensus statement: chromosomal microarray is a first-tier clinical diagnostic test for individuals with developmental disabilities or congenital anomalies. Am J Hum Genet 2010;86: $749-764$.

15. Hochstenbach R, van Binsbergen E, Engelen J, et al. Array analysis and karyotyping: workflow consequences based on a retrospective study of 36,325 patients with idiopathic developmental delay in the Netherlands. Eur $J$ Med Genet 2009;52:161-169.

16. Shen Y, Dies KA, Holm IA, et al. Clinical genetic testing for patients with autism spectrum disorders. Pediatrics 2010;125:e727-e735.

17. Ullmann R, Turner G, Kirchhoff M, et al. Array CGH identifies reciprocal 16 p13.1 duplications and deletions that predispose to autism and/or mental retardation. Hum Mutat 2007;28:674-682.

18. Miller DT, Shen Y, Weiss LA, et al. Microdeletion/duplication at $15 \mathrm{q} 13.2 \mathrm{q} 13.3$ among individuals with features of autism and other neuropsychiatric disorders. J Med Genet 2009;46:242-248.

19. Sebat J, Lakshmi B, Malhotra D, et al. Strong association of de novo copy number mutations with autism. Science 2007;316:445-449.

20. Christian SL, Brune CW, Sudi J, et al. Novel submicroscopic chromosomal abnormalities detected in autism spectrum disorder. Biol Psychiatry 2008; 63:1111-1117

21. Edelmann L, Hirschhorn K. Clinical utility of array CGH for the detection of chromosomal imbalances associated with mental retardation and multiple congenital anomalies. Ann NY Acad Sci 2009;1151:157-166.

22. Sismani C, Kitsiou-Tzeli S, Ioannides M, et al. Cryptic genomic imbalances in patients with de novo or familial apparently balanced translocations and abnormal phenotype. Mol Cytogenet 2008;1:15.

23. Adam MP, Justice AN, Schelley S, et al. Clinical utility of array comparative genomic hybridization: uncovering tumor susceptibility in individuals with developmental delay. J Pediatr 2009;154:143-146.

24. Adams SA, Coppinger J, Saitta SC, et al. Impact of genotype-first diagnosis: the detection of microdeletion and microduplication syndromes with cancer predisposition by aCGH. Genet Med 2009;11:314-322.

25. Rujescu D, Ingason A, Cichon S, et al. Disruption of the neurexin 1 gene is associated with schizophrenia. Hum Mol Genet 2009;18:988-996.

26. Roohi J, Montagna C, Tegay DH, et al. Disruption of contactin 4 in three subjects with autism spectrum disorder. J Med Genet 2009;46:176-182.

27. Moeschler JB. Medical genetics diagnostic evaluation of the child with global developmental delay or intellectual disability. Curr Opin Neurol 2008;21:117-122. 
28. Moeschler JB. Genetic evaluation of intellectual disabilities. Semin Pediatr Neurol 2008;15:2-9.

29. Saam J, Gudgeon J, Aston E, et al. How physicians use array comparative genomic hybridization results to guide patient management in children with developmental delay. Genet Med 2008;10:181-186.

30. Miller DT, Shen Y, Wu B-L. Oligonucleotide microarrays for clinical diagnosis of copy number variation. Curr Protoc Hum Genet 2008;58: 8.12.1-8.12.17.

31. Shearer BM, Thorland EC, Gonzales PR, et al. Evaluation of a commercially available focused aCGH platform for the detection of constitutional chromosome anomalies. Am J Med Genet 2007;143A:2357-2370.

32. Bruno DL, Ganesamoorthy D, Schoumans J, et al. Detection of cryptic pathogenic copy number variations and constitutional loss of heterozygosity using high resolution SNP microarray analysis in 117 patients referred for cytogenetic analysis and impact on clinical practice. J Med Genet 2009;46: $123-131$.

33. McCarroll SA. Extending genome-wide association studies to copy number variation. Hum Mol Genet 2008;17:R135-R142.
34. Van Vooren S, Cossens B, De Moor B, et al. Array comparative genomic hybridization and computational genome annotation in constitutional cytogenetics: suggesting candidate genes for novel submicroscopic chromosomal imbalances syndromes. Genet Med 2007;9:642-649.

35. Lu X, Shaw CA, Patel A, et al. Clinical implementation of chromosomal microarray analysis: summary of 2513 postnatal cases. PLoS One 2007;2: e327.

36. Xiang B, Li A, Valentin D, et al. Analytical and clinical validity of wholegenome oligonucleotide array comparative genomic hybridization for pediatric patients with mental retardation and developmental delay. Am J Med Genet A 2008;146A:1942-1954.

37. Stankiewicz P, Beaudet AL. Use of array CGH in the evaluation of dysmorphology, malformations, developmental delay, and idiopathic mental retardation. Curr Opin Genet Dev 2007;17:182-192.

38. Scott SA, Cohen N, Brandt T, et al. Detection of low-level mosaicism and placental mosaicism by oligonucleotide array comparative genomic hybridization. Genet Med 2010;12:85-92. 